\title{
Multidimensional Attitudes Scale Toward Persons With Disabilities (MAS): tradução e adaptação à língua portuguesa (MAS-PT)
}

\author{
Multidimensional Attitudes Scale Toward Persons With Disabilities (MAS): translation and adaptation to the \\ Portuguese language (MAS-PT)
}

\section{Multidimensional Attitudes Scale Toward Persons With Disabilities (MAS): traducción y adaptación a la lengua portuguesa (MAS-PT)}

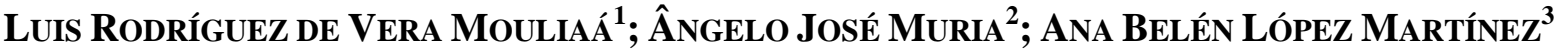 \\ UniVersidade Pedagógica de MAPUTO, UPM, MAPUTO, MoçAMBIQUe \\ Universidad Católica San Antonio de Murcia, UCAM, Murcia, EsPaña
}

\section{RESUMO}

Um dos fatores determinantes para a inclusão social das pessoas com deficiência é a atitude. Entre os instrumentos criados para a sua avaliação encontra-se a Multidimensional Attitudes Scale Toward Persons With Disabilities (MAS), originalmente em língua inglesa. Objetivo: traduzir a escala MAS à língua portuguesa, fazendo a sua adaptação transcultural ao contexto moçambicano. Método: o processo incluiu as etapas de tradução, retrotradução, reunião de peritos e pré-teste. Participaram do pré-teste 24 estudantes (12 de sexo feminino). Resultado: produziu-se uma versão em língua portuguesa da escala MAS (MAS-PT) com uma adequada validade de conteúdo, tendo em conta a equivalência conceitual, semântica, idiomática e experimental. Conclusão: a escala MAS-PT foi satisfatoriamente traduzida e adaptada ao contexto moçambicano, ficando pronta para a avaliação das suas propriedades psicométricas. Palavras-chave: Adaptação Transcultural. Atitudes. Pessoas com Deficiência.

\begin{abstract}
One of the determining factors for the social inclusion of people with disabilities is the attitude. Among the instruments created for its evaluation is the Multidimensional Attitudes Scale Toward Persons With Disabilities (MAS), originally in English. Objective: to translate the MAS scale to the Portuguese language, making its crosscultural adaptation to the Mozambican context. Method: the process included the stages of translation, backtranslation, expert meeting and pre-test. 24 students (12 female) participated in the pre-test. Result: a Portuguese version of the MAS scale (MAS-PT) was produced with adequate content validity, taking into account the conceptual, semantic, idiomatic and experimental equivalence. Conclusion: the MAS-PT scale was satisfactorily translated and adapted to the Mozambican context, being ready for the assessment of its psychometric properties.

Keywords: Cross-Cultural Adaptation. Attitudes. Persons with Disabilities
\end{abstract}

\section{RESUMEN}

Uno de los factores determinantes para la inclusión social de las personas con discapacidad es la actitud. Entre los instrumentos creados para su evaluación se encuentra la Escala de actitudes multidimensionales hacia las personas con discapacidad (MAS), originalmente en inglés. Objetivo: traducir la escala MAS al idioma portugués, haciendo su adaptación intercultural al contexto mozambiqueño. Método: traducción y adaptación transcultural de instrumentos. El proceso incluyó las etapas de traducción, traducción inversa, reunión de expertos y prueba previa. 24 estudiantes (12 mujeres) participaron en la prueba previa. Resultado: se produjo una versión portuguesa de la escala MAS (MAS-PT) con una validez de contenido adecuada, teniendo en cuenta la equivalencia conceptual, semántica, idiomática y experimental. Conclusión: la escala MAS-PT se tradujo satisfactoriamente y se adaptó al contexto mozambiqueño, estando lista para la evaluación de sus propiedades psicométricas.

Palabras clave: Adaptación Transcultural. Actitudes. Personas con Discapacidad.

\footnotetext{
1 Professor do Departamento de Socio-Antropometria da UPM. E-mail: fumoam@hotmail.com. ORCID: http://orcid.org/0000-0003-0478-3623.

2 Professor do Departamento de Socio-Antropometria da UPM. E-mail: ajmuria@gmail.com. ORCID: http://orcid.org/0000-0003-0563-9068.

Professora do Departamento de Educação Física da UCAM. E-mail: almartinez@ucam.edu. ORCID: http://orcid.org/0000-0001-8419-3599.
} 


\section{INTRODUÇÃO}

Atualmente, o termo "inclusão" tem sido muito utilizado em contextos tão diversos como o educativo, cultural, desportivo, digital e financeiro, entre outros. Independentemente do âmbito, quando utilizado para referir-se às pessoas com deficiência, adverte-se que a maior parte das barreiras encontradas está relacionada com as atitudes sociais (KROPP; WOLFE, 2018; OMOTE, 2018; TANAKA, 2007; XAVIER, 2016). Se no entorno das pessoas com deficiência as atitudes forem positivas, haverá espaço para a inclusão; pelo contrário, quando as atitudes forem negativas, poderão dificultar este processo, convertendo deficiências específicas em desvantagens pessoais, familiares e profissionais (FINDLER; VILCHINSKY; WERNER, 2007).

Portanto, um dos fatores chave para a inclusão social das pessoas com deficiência será a atitude das pessoas sem deficiência perante este grupo. Segundo Rao (2004, como citado por FINDLER; VILCHINSKY; WERNER, 2007), apesar de não existir um consenso universal entre as mais de 30 definições da atitude, os pesquisadores parecem estar de acordo em incluir três dimensões: cognitiva, afetiva e comportamental (OCETE; PÉREZ-TEJERO; COTERÓN, 2015; TANAKA，2007; VILCHINSKY; WERNER; FINDLER, 2010; e outros).

Neste contexto, o conhecimento, pensamentos e crenças que as pessoas têm em relação à deficiência, isto é, a dimensão cognitiva, tende a estimular certos sentimentos ou emoções no indivíduo, ou seja, a dimensão afetiva. Como consequência destas convicções e sentimentos provocados, ao entrar em contato com uma pessoa com deficiência, o sujeito tende a atuar de uma determinada maneira manifestando assim a dimensão comportamental da atitude (KIM; LU; ESTRADA-HERNANDEZ, 2015; RADLIŃSKA et al., 2020; VILCHINSKY; WERNER; FINDLER, 2010). Portanto, desde uma perspectiva multidimensional, considera-se a atitude como um conjunto duradouro de crenças, carregadas de emoção que predispõe uma pessoa para determinados tipos de comportamentos (SHERRILL, 1998). Nestes termos, pode-se deduzir que o comportamento de uma pessoa parece ser o resultado do que pensa e sente, e portanto, para induzir um comportamento positivo, deveria influenciar-se sobre essas duas componentes (OCETE; PÉREZ-TEJERO; COTERÓN, 2015; RADLIŃSKA et al., 2020).

As pesquisas sobre a inclusão das pessoas com deficiência indicam que existe uma necessidade de aumentar a consciencialização da sociedade sobre a deficiência e superar a imagem estereotipada construída a partir dos preconceitos (OMOTE, 2018). Desta forma, se faz necessário que sejam avaliadas cuidadosamente as atitudes em relação às pessoas com deficiência para a definição de metas e planos de atuação (PALAD et al., 2016). Neste sentido, grande parte dos pesquisadores tem usado questionários de avaliação em diferentes âmbitos como instrumento para a recolha $\mathrm{e}$ registo de dados de forma sistemática e cuidadosa (RAMOS, 2014).

Como resultado da revisão da literatura (KIM; LU; ESTRADA-HERNANDEZ, 2015; KROPP; WOLFE, 2018; MARTÍN; ARREGUI, 2013; OMOTE, 2018; PALAD et al., 2016; RADLIŃSKA et al., 2020; WÖHRLE; FRANKE; KISSGEN, 2018), concluiu-se que o questionário intitulado Multidimensional Attitudes Scale Toward Persons With Disabilities - MAS, construído e validado por Findler, Vilchinsky e Werner (2007) e revalidado pelas mesmas autoras em Vilchinsky, Werner e Findler (2010), permitia uma aproximação multidimensional ao conceito da atitude ora apresentado, tendo excelente validade e consistência interna. No entanto, como ocorre em muitas ocasiões, os questionários são utilizados em línguas e contextos diferentes daqueles para os quais foram originariamente criados. Assim, para que mantenham a 
sua equivalência e possam apresentar resultados comparáveis dentro da comunidade científica, não basta realizar uma tradução direta, sendo necessária uma adaptação e validação ao novo contexto cultural onde será utilizado (BERTOLAZI, 2008; FERREIRA et al., 2014; FORTES; ARAÚJO, 2019; MENDONÇA et al., 2018). Neste sentido, a adaptação transcultural, deve combinar a tradução literal do instrumento e a sua acomodação contextual e cultural à população alvo (BEATON et al., 2007; COSTER; MANCINI, 2015; DORTAS JUNIOR et al., 2016).

Em Moçambique, o último censo geral da população ${ }^{4}$, realizado no ano 2017 pelo Instituto Nacional de Estatística, estimou que 2,6\% da população tinha algum tipo de deficiência. Face isto, o Governo de Moçambique tem colocado entre as suas prioridades e desafios a inclusão deste grupo social, desenvolvendo um quadro legislativo e orgânico oficial através de leis, decretos, estratégias, planos de ação etc. Porém persiste uma grave estigmatização deste grupo, que é manifestada através do seu fraco acesso à educação, saúde, atividade profissional, recreação, atividades desportivas, e outras (MINGANE; RELANDEAU; WAMUSSE, 2010; NHAQUILA, 2011; SIGAÚQUE, 2013; entre outros). O Plano Nacional de Ação para Área da Deficiência (PNAD) indica que "[...] ainda persistem algumas atitudes negativas por parte das famílias, das comunidades e da sociedade em geral, em relação a este segmento da sociedade, que concorrem para a sua exclusão social e discriminação" (MOÇAMBIQUE, 2012, p. 12). A este respeito, Radlińska et al. (2020) afirmam que a eliminação de barreiras ambientais e organizacionais parece bem mais simples de implementar do que a eliminação real das atitudes sociais negativas.

Neste âmbito, adverte-se a inexistência de um questionário adaptado ao contexto moçambicano que aborde uma avaliação da atitude perante a pessoa com deficiência desde uma perspectiva multidimensional. Portanto, o presente estudo teve como objetivo traduzir para a língua portuguesa e adaptar ao contexto moçambicano a escala Multidimensional Attitudes Scale Toward Persons With Disabilities (MAS), originalmente desenvolvida na língua inglesa.

\section{MÉTODo}

O presente estudo faz parte de um trabalho maior, que está sendo realizado em duas etapas: a etapa 1, refere-se à tradução e adaptação cultural; enquanto que a etapa 2 é relativa à avaliação das propriedades psicométricas do questionário a partir da análise estatística. Neste sentido, para elaboração do presente artigo recorre-se somente a primeira etapa do estudo. Para o início da pesquisa e tendo em consideração as questões éticas e respeito da autoria do instrumento a ser adotado, foi necessário contatar as autoras do questionário original. Do contato resultou a autorização das autoras no sentido do questionário MAS passar por um processo de tradução para língua portuguesa e a sua adaptação cultural no contexto moçambicano.

Dentre os métodos usados na literatura para a tradução e adaptação cultural, utilizou-se a guia metodológica proposta por Beaton et al. (2002; como citados em FERREIRA et al., 2014) e apoiada pela American Academy of Orthopedic Surgeons/Institue of Work and Health (AAOS/IWH), que tem sido usada internacionalmente por pesquisadores de diversas áreas como a medicina, sociologia e psicologia (BRACCIALLI et al., 2019a; BRACCIALLI et al., 2019b; COSTER; MANCINI, 2015; FERREIRA et al., 2014; MENDONÇA et al., 2018). De acordo com

\footnotetext{
${ }^{4}$ Disponível em: http://www.ine.gov.mz/iv-rgph-2017/mocambique/censo-2017-brochura-dos-resultadosdefinitivos-do-iv-rgph-nacional.pdf. Acesso em: 10 mar. 2020.
} 
a guia, o uso de questionários auto-administráveis em países, culturas e/ou idiomas diferentes às originais do instrumento precisa de um tratamento metodológico que permita alcançar equivalências na tradução e adaptação entre o instrumento original e o idioma alvo a ser traduzido. Assim, os autores recomendam cinco passos para a tradução e adaptação cultural de instrumentos (FERREIRA et al., 2014, p. 458): i) traduções independentes; ii) síntese das traduções; iii) retrotraduções da síntese; iv) reunião de um comitê de peritos; v) pré-teste.

Figura 1: Descrição dos passos previstos na guia AAOS/IWH, com acréscimo das modificações sugeridas (em itálico).

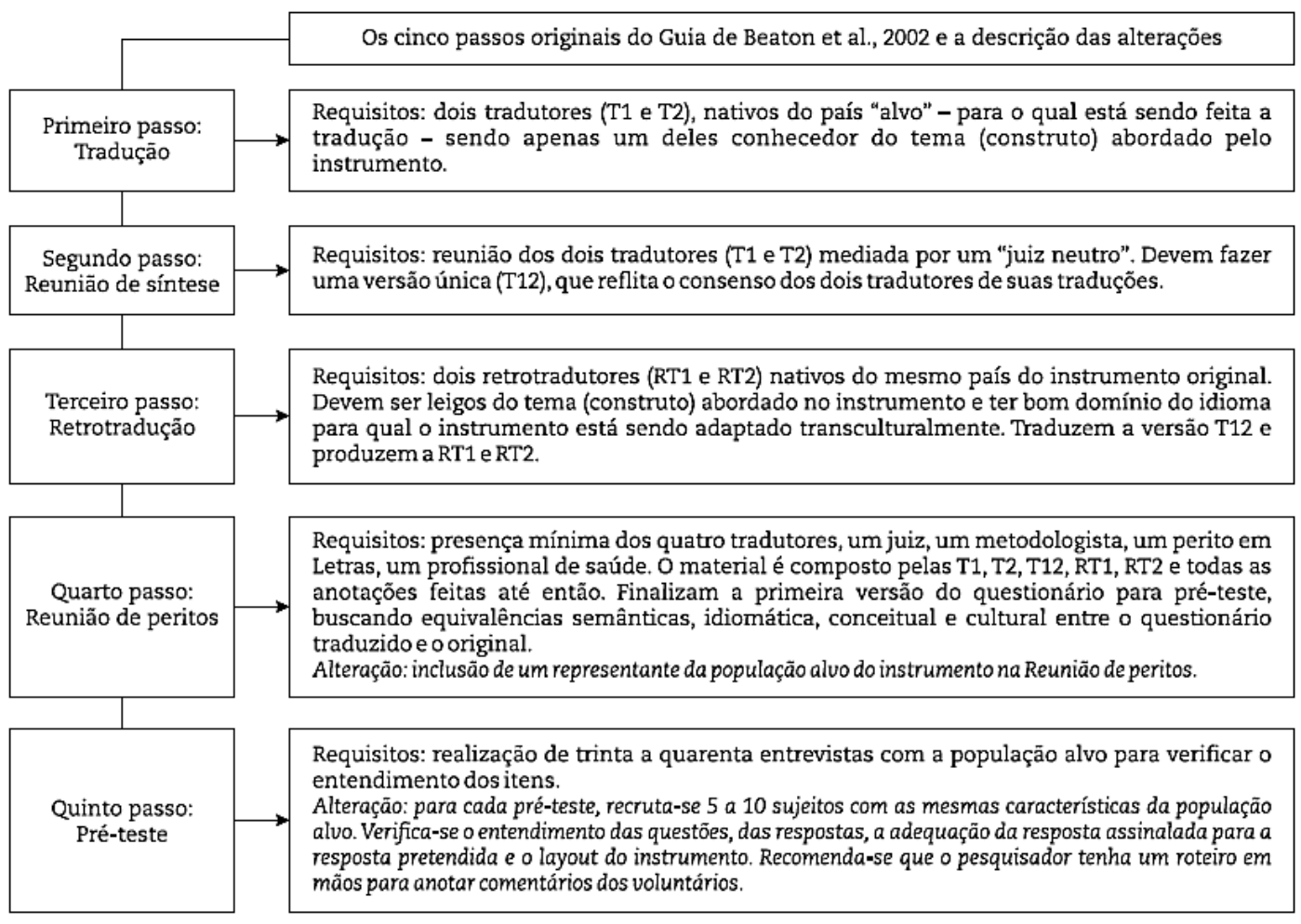

Fonte: Ferreira et al. (2014, p. 460).

Apesar da clareza e sequência lógica do processo, Ferreira et al. (2014), acrescentaram duas alterações que trouxeram alguma melhoria na qualidade da tradução e adaptação cultural (Figura 1). A primeira alteração foi realizada no quarto passo com a agregação de um representante da população alvo à reunião de peritos. Entre as vantagens, podem-se destacar: o retorno imediato sobre a percepção de cada item por parte dos destinatários finais do questionário; maior qualidade e coerência do trabalho; diminuição do número de questionamentos feitos pelos participantes durante os prétestes; e processo de adaptação mais célere e, portanto, mais econômico.

A segunda alteração foi efetuada no quinto passo estando relacionada com a redução do número de participantes durante o pré-teste, sendo recomendada uma amostra de cinco a dez sujeitos (também apoiada por BRACCIALLI et al., 2019b). Os autores indicam que a entrevista feita durante o pré-teste a cada um dos participantes após o preenchimento do questionário requer concentração do pesquisador, tempo e disposição dos participantes. Neste sentido, um grupo menor de participantes favorece a qualidade do processo. Por razões de interesse relacionadas às características do instrumento, na presente pesquisa aumentou-se para 12 o número de sujeitos em cada 
grupo de pré-testagem. Portanto, a Figura 1 resume os passos que foram seguidos para a tradução e adaptação cultural do questionário MAS neste estudo.

\section{CARACTERÍSTICAS DOS PARTICIPANTES}

O processo de tradução e adaptação cultural do questionário envolveu uma série de participantes, que por questões éticas, tiveram que assinar um Termo de Consentimento Livre e Esclarecido. No termo, foi explicado que o interesse não residia especificamente nas respostas ao instrumento, e sim no processo de preenchimento e entrevista subsequente. $\mathrm{O}$ quadro 1 apresenta as características dos participantes em cada um destes passos.

Quadro 1: Características dos participantes em cada um dos passos da pesquisa.

\begin{tabular}{|c|c|c|}
\hline Passo & Objetivo & Participantes \\
\hline Passo I & $\begin{array}{l}\text { Tradução do } \\
\text { instrumento }\end{array}$ & $\begin{array}{l}\text { - Dois tradutores de nacionalidade moçambicana, ambos licenciados } \\
\text { em língua inglesa. }\end{array}$ \\
\hline Passo II & $\begin{array}{l}\text { Reunião de } \\
\text { síntese }\end{array}$ & $\begin{array}{l}\text { - Os dois tradutores do passo I. } \\
\text { - Um juiz, de nacionalidade moçambicana, licenciado em língua } \\
\text { portuguesa. }\end{array}$ \\
\hline Passo III & Retrotradução & $\begin{array}{l}\text { - Um tradutor de nacionalidade israelita, residente em Moçambique } \\
\text { por mais de quatro anos e fluente na língua portuguesa. } \\
\text { - Um tradutor de nacionalidade inglesa, residente em Moçambique } \\
\text { por mais de três anos e fluente na língua portuguesa. }\end{array}$ \\
\hline Passo IV & $\begin{array}{l}\text { Reunião de } \\
\text { peritos }\end{array}$ & $\begin{array}{l}\text { - Todos os participantes dos passos II e III. } \\
\text { - Um especialista na área da deficiência, com pós-graduação na área } \\
\text { de educação. } \\
\text { - Um pesquisador com experiência na adaptação cultural de } \\
\text { questionários, com doutorado em biologia marinha. } \\
\text { - Um estudante universitário no curso de Educação Física. }\end{array}$ \\
\hline \multirow{2}{*}{ Passo V } & Pré-teste I & $\begin{array}{l}\text { - Seis participantes de sexo feminino (entre } 19 \text { e } 28 \text { anos). Todos } \\
\text { estudantes: quatro do ensino superior e dois com } 12^{\text {a }} \text { Classe. } \\
\text { - Seis participantes de sexo masculino (entre } 21 \text { e } 30 \text { anos). Todos } \\
\text { estudantes: quatro do ensino superior e dois da } 11^{\text {a }} \text { Classe }^{5} \text {. }\end{array}$ \\
\hline & Pré-Teste II & $\begin{array}{l}\text { - Seis participantes de sexo feminino (entre } 18 \text { e } 29 \text { anos). Todos } \\
\text { estudantes: quatro do ensino superior e dois da } 11^{\text {a }} \text { Classe. } \\
\text { - Seis participantes de sexo masculino (entre } 21 \text { e } 33 \text { anos). Todos } \\
\text { estudantes: quatro do ensino superior e dois da } 12^{\text {a }} \text { Classe. }\end{array}$ \\
\hline
\end{tabular}

Fonte: Elaboração própria.

\section{O QUESTIONÁRIO MAS}

A escala MAS foi criada e validada por Findler, Vilchinsky e Werner (2007) para a população israelita, com o intuito de avaliar as atitudes das pessoas sem deficiência perante as pessoas com deficiência através dos três domínios: cognitivo, emocional e comportamental. Cada domínio foi representado por uma subescala que pontua para o somatório total. A escala inicia descrevendo uma situação fictícia onde uma pessoa sem deficiência - que pode ser um homem (José) ou mulher (Michela) - saiu para almoçar com alguns amigos. Durante o almoço uma pessoa em cadeira de rodas (homem ou mulher) une-se ao grupo. Após os seus amigos saírem, apenas ficam o José/Michela (esperando pela sua boleia/carona) e o homem/mulher de cadeira de rodas. Neste contexto, o inquerido é convidado a colocar-se no lugar do José/Michela, e indicar as emoções, pensamentos e comportamentos que ele/ela poderia experimentar. A situação

\footnotetext{
${ }^{5}$ Ensino secundário. Para mais informações, consultar: http://www.mined.gov.mz/EDUCA/Pages/EnsinoSecund\%C3\%A1rio-Geral.aspx. Acesso em: 10 mar. 2020.
} 
descrita anteriormente, está montada num cenário social que permite ao respondente, de forma indireta, projetar-se na personagem e expressar com sinceridade a sua opinião (VILCHINSKY; WERNER; FINDLER, 2010).

O questionário MAS está composto por 34 perguntas divididas em três dimensões: afetiva (16 perguntas, sendo 11 reações emotivas negativas e quatro reações emotivas positivas); cognitiva (10 perguntas) e comportamental (8 perguntas). Todas as questões são respondidas numa escala Likert de cinco pontos, sendo 1 ("para nada") e 5 ("totalmente"). Para a análise dos resultados deve ter-se em conta que os escores mais baixos indicam uma atitude mais positiva perante a deficiência.

\section{PROCEDIMENTOS PARA A TRADUÇÃO E ADAPTAÇÃO}

De acordo com as recomendações feitas na literatura específica (BEATON et al., 2007; BRACCIALLI et al., 2019a; COSTER; MANCINI, 2015; MENDONÇA et al., 2018; REICHENHEIM; MORAES, 2007) e seguindo as alterações propostas por Ferreira et al. (2014), o processo de tradução e adaptação cultural da escala MAS, foi realizado em 5 passos metodológicos:

\section{PRIMEIRO PASSO: TRADUÇÃO}

A tradução inicial da língua inglesa para a língua portuguesa, foi efetuada de forma independente por dois tradutores de nacionalidade moçambicana, ambos licenciados em língua inglesa, sem relação entre eles e com setores de trabalho diferentes, sendo que a um deles foi-lhe facilitada informação adicional sobre a atitude e as suas dimensões.

Entre as orientações que foram dadas aos tradutores estavam: realização de uma tradução clara e compreensível; uso de uma linguagem simples e de fácil entendimento para um grupo populacional amplo; privilegiar a equivalência conceitual em detrimento da tradução literal e evitar um duplo negativo. Portanto, foi produzida uma tradução da escala original para a língua portuguesa por cada um deles, resultando em duas versões (T1 e T2).

\section{SEGUNDO PASSO: REUNIÃO DE SÍNTESE}

O trabalho realizado por cada tradutor no passo anterior foi partilhado durante um encontro no qual participaram, além de ambos tradutores, um especialista em língua portuguesa, a título de "mediador". O propósito principal da reunião foi a obtenção de um consenso em relação às traduções, devendo o especialista em língua portuguesa elaborar uma versão única da escala MAS traduzida ao português (T12).

O processo de conciliação foi realizado item por item, sendo escolhida a melhor tradução em cada caso, ou sugerindo uma nova tradução se não houvesse consenso. Neste sentido, o grupo avaliou a equivalência conceitual (algumas palavras podem ter um significado conceitual diferente entre as culturas), a equivalência semântica (as palavras devem ter o mesmo significado em ambas línguas; ou deve ser escolhido o melhor significado no caso de apresentarem mais do que um; e ainda identificar se existem dificuldades gramaticais na tradução) e a equivalência idiomática (coloquialismos e expressões idiomáticas difíceis de traduzir requerem uma "expressão equivalente" que não modifique o sentido cultural original).

\section{TERCEIRO PASSO: RETROTRADUÇÃO}

Foram realizadas duas traduções independentes para a língua inglesa da versão conciliada T12. Foram contatados dois tradutores para o efeito: o primeiro de origem israelita, país original do instrumento; e o segundo de origem inglesa, ambos residentes 
em Moçambique por mais de três anos e fluentes na língua portuguesa. Nenhum dos tradutores conheciam o questionário MAS, ou estavam familiarizados com aspectos específicos relacionados ao conceito de atitude ou deficiência. Estes tradutores elaboraram as retrotraduções 1 e 2 (RT1 e RT2).

\section{QUARTO PASSO: REUNIÃO DE PERITOS}

Foram convidados para uma reunião todos os intervenientes nos passos anteriores (os quatro tradutores e o mediador), um especialista na área da deficiência, um pesquisador em qualidade de metodologista com experiência na adaptação cultural de questionários, e um estudante universitário sem deficiência. Com a inclusão deste último membro pretendia-se ter um representante da população alvo já que a escala MAS está dirigida a pessoas sem deficiência.

Durante o encontro, foi dada informação aos participantes sobre o processo de tradução e adaptação cultural do instrumento, ademais de fornecerem-se o questionário original e todas as versões (T1, T2, T12, RT1, RT2) e anotações de cada passo. Partindo da versão de consenso em língua portuguesa (T12) e sua comparação com as retrotraduções (RT1 \& RT2), todos os participantes foram orientados a contribuir com opiniões para cada um dos itens da escala. As decisões tomadas nesta reunião deviam garantir que entre a escala original e a traduzida existisse equivalência semântica, conceitual, idiomática e experimental (os itens do instrumento devem ser aplicáveis na nova cultura ou substituídos por um item equivalente). Como resultado deste encontro, foi elaborada a segunda versão do questionário MAS em língua portuguesa, e que foi identificada como MAS-PT ${ }^{6}$.

\section{QUINTO PASSO: PRÉ-TESTAGEM}

O quinto passo foi desenvolvido a partir de três momentos: contato do pesquisador com os participantes da amostra (preenchimento da escala MAS-PT e realização de uma entrevista semiestruturada de forma individual), compilação dos dados recolhidos e trabalho de consenso no grupo de peritos.

Inicialmente, foram escolhidos 12 sujeitos (seis de cada sexo) de forma intencional, para a realização da pré-testagem. De acordo às autoras da escala original (FINDLER; VILCHINSKY; WERNER, 2007), os sujeitos de sexo masculino receberam a escala referente ao "José" e as participantes de sexo feminino receberam-na referente à "Michela". Na metade das escalas a pessoa em cadeira de rodas era um homem e na outra metade era uma mulher. Neste sentido, foram elaboradas quatro versões (sexo da personagem vs. sexo da pessoa em cadeira de rodas), obtendo os dados de três participantes para cada uma das versões.

Para facilitar a recolha de dados e evitar a perda de informação importante foi desenhado um roteiro de entrevista. Foram elaboradas também questões para verificar o grau de entendimento de cada participante em relação ao conteúdo da cena inicial e das perguntas e respostas para cada unidade de conteúdo (p. ex.: sentiu dificuldades em compreender este item? poderia sugerir outra forma de escrever sem perder o sentido? existe algum termo que não percebe? é fácil de responder? etc.); assim como foi conferida a resposta escolhida pelo participante em relação à resposta desejada. Igualmente, foi verificado o parecer dos entrevistados relativamente ao formato do questionário, tipo de letra, tamanho da fonte etc.

Após a compilação dos dados recolhidos, foi novamente reunido o grupo de peritos (estando ausentes um tradutor e um retrotradutor por motivos justificados).

\footnotetext{
${ }^{6}$ Pela sigla de Portugal (PT).
} 
Neste novo encontro, foram analisados os dados recolhidos durante as entrevistas, primeiramente de forma global (respostas de cada sujeito), e em seguida de forma individualizada (análise de cada unidade de conteúdo), após o qual foi decidido realizar algumas modificações. Deste processo resultou uma segunda versão do questionário MAS-PT, a qual seguiu o mesmo procedimento da versão anterior, sendo aplicada a escala e realizadas entrevistas a um novo grupo de 12 sujeitos.

Os dados coletados foram novamente compilados para melhor compreensão e submetidos para a sua análise ao conjunto de peritos (estando ausente um tradutor por motivo justificado). Uma vez confirmada a ausência de informações relevantes que justificassem uma nova versão foi decidido, por unanimidade, finalizar o processo de tradução e adaptação da escala MAS-PT.

\section{APRESENTAÇÃo dOS RESUlTAdOS}

O processo de tradução e adaptação cultural permitiu reunir a vários profissionais de diferentes áreas de conhecimento, assim como um grupo de 24 participantes para a pré-testagem do instrumento divididos em dois grupos. Os resultados foram apresentados em três momentos: 1) reunião de síntese; 2) reunião de peritos; 3) préteste.

\section{1) REUNIÃO DE SÍNTESE}

O processo de síntese foi enriquecido graças às versões elaboradas pelos tradutores, as quais mesmo similares entre si, trouxeram diferentes formas de interpretação. Com o intuito de produzir uma versão consensual na língua portuguesa (T12), o questionário foi dividido em 62 unidades de conteúdo sem detrimento da visão holística do texto. Como resultado da reunião de síntese, foram aceites 10 traduções do tradutor 1 e 22 do tradutor 2. Em 23 ocasiões houve consenso entre ambos tradutores e sete unidades de conteúdo foram reelaboradas e tidas como "novas traduções".

Neste contexto, foi possível constatar uma diferença entre o número de contribuições de cada tradutor (10 vs. 22) que pode ser justificada pela informação adicional facilitada ao tradutor 2 em relação à atitude e suas dimensões. Ainda, a função do mediador especialista em língua portuguesa foi tida como uma mais-valia para o processo de equivalência, já que a conciliação de traduções permitiu a reelaboração de algumas unidades de conteúdo para facilmente serem entendidas pela população alvo deste estudo (p. ex.: na unidade de conteúdo 23 trocou "desamparo" por "abandono"; ou na unidade de conteúdo 27 trocou "serenidade" por "tranquilidade").

Finalmente, apesar de ter-se alcançado um consenso na tradução em todas as unidades de conteúdo, foram registados alguns pontos que mereceriam de uma revisão na reunião de peritos (p. ex.: eliminação da palavra vignette, traduzida como "cenário"; o uso da palavra "afeto"; ou a utilização do termo "cognição").

\section{2) REUNIÃO DE PERITOS}

Durante a comparação entre retrotraduções, foi advertido que das 62 unidades de conteúdo, 34 foram idênticas entre si, e 28 foram semelhantes. Quando conferida a similitude entre as retrotraduções e a escala original, foi verificado que a retrotradução 1 continha 19 unidades de conteúdo idênticas ao instrumento original, e a retrotradução 2 partilhava 14 unidades de conteúdo idênticas ao questionário original. No entanto, as unidades de conteúdo não consideradas como idênticas, foram consideradas como semelhantes. 
Assim, cada uma das 62 unidades de conteúdo, foram examinadas pela reunião de peritos em base às equivalências semânticas, conceituais, idiomáticas e experimentais, com o intuito de produzir uma versão de consenso. A seguir foram arroladas as principais mudanças levadas a cabo como resultado desta análise:

Na unidade de conteúdo 1, foi decidido colocar a abreviatura "MAS-PT" no título da escala. Embora sendo uma tradução e adaptação transcultural à espera de um procedimento estatístico de validação, foi considerado adequado identificar e diferenciar a versão na língua portuguesa da escala original em inglês.

Como sugerido na reunião de síntese, foi decidido retirar a palavra "cenário" (no original vignette) da unidade de conteúdo 2 , por ser considerada desnecessária já que a primeira linha do texto da unidade de conteúdo seguinte introduzia o leitor na cena inicial: "Imagine a seguinte situação". Ainda na unidade de conteúdo 3, o ponto-final na frase original, foi substituído por dois-pontos, tendo em conta que o sentido da frase era introduzir o leitor num cenário particular. Igualmente, as aspas no início desta mesma unidade de conteúdo, foram deslocadas até no início da unidade de conteúdo 4 , entendendo que as aspas são um sinal de pontuação cuja principal finalidade é destacar alguma parte de um texto, distinguindo-a do restante, neste caso o cenário inicial.

$\mathrm{Na}$ unidade de conteúdo 4, foi decidido trocar o termo "cafetaria" (do original coffee shop), para "restaurante". Isto foi devido a que o termo cafetaria faz referência ao lugar onde se bebe café, não sendo esta uma bebida de consumo frequente em Moçambique. Além disso, mesmo sendo relativamente formal o termo "restaurante" e tendo em conta que as pessoas de baixa renda no país não têm o hábito de frequentá-los, foi considerado como de fácil compreensão, ajudando a situar o cenário inicial.

Na unidade de conteúdo 10, "todo o mundo sai" (do original everyone else leaves) foi mudado para "todos os outros vão embora". Para isto, foi tido em conta que a expressão "ir embora" (proveniente de "ir em+boa+hora") é usualmente utilizada em Moçambique e reflete a ideia das pessoas terem ido do local definitivamente. A ideia da tradução inicial o verbo sair poderia indicar que as pessoas ausentaram-se mas seria possível que voltassem novamente (p. ex.: "ele saiu... mas volta já").

$\mathrm{Na}$ unidade de conteúdo 15, foi determinado pelo grupo de peritos, trocar " $\mathrm{Na}$ seguinte coluna, está uma lista..." (do original In the next column is a list...) para "À direita está uma lista...". De acordo ao formato original da escala, foi percebido que esta mudança melhorava a localização da tabela no espaço da folha facilitando assim a compreensão do leitor.

Na unidade de conteúdo 18, o termo "afeto" (do original affect), foi substituído por "emoção" devido a duas razões. Primeira, foi pretendido evitar a distração do respondente com a introdução de um novo conceito, já que no parágrafo precedente (unidades de conteúdo 14, 15 \& 16) foi utilizado o termo "emoção" (emotion) em três ocasiões, mas nenhuma o termo "afeto" (affect). Portanto, foi uniformizado o termo usado com o intuito de facilitar a compreensão do leitor. Segunda, parece que as autoras do questionário original, ao longo do seu artigo, tratam indistintamente ambos os termos (affect \& emotion) para referir-se ao mesmo conceito (FINDLER; VILCHINSKY; WERNER, 2007).

Nas unidades de conteúdo 23 e 27, foi decidido manter a mudança realizada durante a reunião de consenso da primeira versão. Em consequência, foram aceites os termos "abandono" (pelo original helplessness) e "tranquilidade" (pelo original serenity), por serem considerados como termos equivalente a nível semântico e mais acessíveis para a população.

$\mathrm{Na}$ unidade de conteúdo 37, o termo "cognições" (do original cognitions), foi trocado para "pensamentos" devido a dois motivos: por um lado, o termo "cognição" foi 
considerado de uso frequente nas áreas da educação e psicologia, mas de difícil compreensão para o público no geral; e por outro lado, o pensamento é uma função cognitiva que forma parte do processo da cognição (capacidade de assimilar e processar a informação recebida do meio interno e externo para construir conhecimento). Portanto, sob esta forte relação, "pensamento" foi escolhido como um termo de uso mais frequente e de fácil entendimento.

Similarmente, foi usado o verbo "pensar" pelas autoras do instrumento original durante a explicação sobre o modo de preenchimento da escala, afirmando que o respondente deveria indicar em que medida acreditavam que o item refletia "[...] a maneira como a pessoa na história (José ou Michela) se sentiria, pensaria ou atuaria nessa situação" (FINDLER; VILCHINSKY; WERNER, 2007, p. 169). Ainda, o mesmo artigo refere que a componente cognitiva da atitude foi retirada de um teste que mede os pensamentos sobre as interações sociais entre pessoas com e sem deficiência, considerando tanto a valência dos pensamentos (positivos ou negativos) quanto o foco da atenção (FINDLER; VILCHINSKY; WERNER, 2007).

Neste contexto, foi determinado pela reunião de peritos que a uniformização dos termos no texto introdutório e na própria tabela, evitaria a distração dos respondentes em aspectos conceituais e aumentaria a atenção para os itens. Como resultado, foi decidido estender esta alteração, "cognição" por "pensamento", para as unidades de conteúdo 39 e 40.

$\mathrm{Na}$ unidade de conteúdo 48, foi alterada a tradução "Eu posso fazê-lo/a sentir-se mais confortável" (do original I can make him/her feel more comfortable) para "Eu posso fazê-lo/a sentir-se mais à vontade". Embora, tanto a tradução (T12) como as retrotraduções (RT1 \& RT2) e o original coincidiram, foi sugerido pelo grupo de peritos o interesse de simplificar a linguagem a fim de ser compreendido pelo maior número de respondentes, mas sem perder a equivalência semântica.

$\mathrm{Na}$ unidade de conteúdo 60, foi modificada a tradução "Trocar de mesa" (do original Move to another table) para "Ir para outra mesa". Portanto, foi considerado que a nova tradução exprimia melhor a intencionalidade no comportamento do protagonista de abandonar a mesa.

\section{3) PRÉ-TESTE}

A versão produzida pela reunião de peritos foi testada em 12 sujeitos. Cada participante preencheu o questionário e seguidamente realizou uma entrevista. Os respondentes demoraram entre quatro e sete minutos para preencher o questionário, e entre 24 e 31 minutos em realizar as entrevistas.

Dos dados coletados foi possível advertir que, quase a totalidade dos sujeitos, teve uma boa compreensão tanto da situação inicial como dos termos utilizados, respondendo positivamente às perguntas da entrevista. Contudo, foram apresentadas dúvidas por seis dos participantes, na unidade de conteúdo 12 ("José/Michela deve esperar 15 minutos pela sua boleia"), indicando dúvida sobre quem devia dar boleia à personagem: uma terceira pessoa ou o próprio leitor. Neste sentido, com o intuito de evitar a ambiguidade, foi substituído o pronome possessivo para a terceira pessoa (no caso "sua"), pela contração "dele/dela" a fim de cumprir uma função semelhante. Portanto, a unidade de conteúdo 12 ficou traduzida como "José/Michela deve esperar 15 minutos pela boleia dele/dela".

Outra inquietação por parte de vários dos respondentes foi tida em relação à forma de preenchimento do questionário, não sabendo se deviam colocar um " $x$ ", riscar, ou colocar um círculo sobre a resposta desejada. Em Moçambique, é habitual que os questionários tenham uma breve explicação introdutória sobre a forma de 
preenchimento do instrumento, sendo comumente necessário marcar com " $\mathrm{x}$ " a resposta correta. Neste sentido, foi decidido aumentar uma breve indicação sobre a forma de preenchimento nas unidades de conteúdo 16, 39 e 53, passando da tradução inicial "Por favor, classifique em cada linha a probabilidade..." para "Por favor, assinale com " $\mathrm{x}$ " em cada uma das 16 linhas para indicar a opção que melhor classifique a probabilidade...". Intencionalmente foi indicado o número total dos itens a pontuar (16, $10 \& 8$, segundo a dimensão), pois vários dos respondentes manifestaram dúvidas no preenchimento, tendo escolhido apenas alguns itens que consideravam aplicáveis à personagem e deixado sem responder aqueles que achavam que não se aplicavam à mesma. Em qualquer caso, esta modificação somente fazia referência à forma de preenchimento, não alterando o conteúdo principal do instrumento.

Em relação ao formato do instrumento, foi decidido alterar o instrumento original e aumentar linhas de separação entre itens dado que alguns dos participantes apresentaram dificuldades para escolher a linha adequada durante a pontuação, e em certas ocasiões, marcando duas vezes sobre o mesmo item.

Os dados compilados na pré-testagem foram objeto de uma nova revisão por parte dos peritos, resultado da qual foi elaborada uma segunda versão do questionário. Esta versão foi testada por um novo grupo de 12 sujeitos, sem relação com o anterior, o qual seguiu o mesmo procedimento do grupo inicial: cada participante preencheu $\mathrm{o}$ questionário e, seguidamente, foi realizada a entrevista. Nesta ocasião, os respondentes dedicaram entre quatro e seis minutos para preencher o questionário, e entre 25 e 29 minutos em realizar as entrevistas.

A partir do segundo pré-teste foi verificado que as questões anteriores tinham sido ultrapassadas, deixando a escala compreensível no contexto moçambicano, sem perder a equivalência semântica, conceitual, idiomática e experimental em relação ao instrumento original. Finalmente, foi dado por encerrado o processo de tradução à língua portuguesa e adaptação cultural ao contexto moçambicano da escala MAS-PT (em apêndice).

\section{DISCUSSÃO}

A escala Multidimensional Attitudes Scale Toward Persons With Disabilities (MAS), criada por Findler, Vilchinsky e Werner (2007) tem sido considerada como uma ferramenta adequada, com excelente consistência interna e boa validade, que mede as atitudes perante as pessoas com deficiência desde uma perspectiva multidimensional (cognitiva, afetiva e comportamental). Ainda o instrumento pode ser adaptado a diferentes grupos sociais, assim como a vários tipos de deficiência (KROPP; WOLFE, 2018; LUND; SEEKINS, 2014; MARTÍN; ARREGUI, 2013; VILCHINSKY; WERNER; FINDLER, 2010).

Neste contexto, o questionário MAS ora traduzido para a língua portuguesa e adaptado ao contexto moçambicano, já foi traduzido e validado em diferentes idiomas como o inglês (original), polaco, alemão, espanhol, coreano, sérvio, turco e francês. Em consequência, foram determinadas as potencialidades da escala como ferramenta na avaliação preliminar e seguimento de programas educacionais e políticos, assim como na comparação de dados entre populações (KIM; LU; ESTRADA-HERNANDEZ, 2015; RADLIŃSKA et al., 2020).

Embora existindo diferenças entre os passos para a realização do processo de tradução e adaptação de um instrumento para uma cultura diferente da qual foi validado (BEATON et al., 2007; BRACCIALLI et al., 2019b; COSTER; MANCINI, 2015; 
FORTES; ARAÚJO, 2019; REICHENHEIM; MORAES, 2007), a maior parte dos autores coincide na necessidade de cumprir uma série de etapas predeterminas que devem ser executadas de forma metódica, tais como: (1) tradução para a língua pretendida; (2) reunião para conciliar as traduções; (3) retrotradução para a língua original; (4) reunião de peritos para a análise de equivalência de itens; e (5) pré-teste. Neste sentido, foi partilhado o rigor científico com a bibliografia, através da apresentação dos dados deste estudo durante a realização de cada um dos passos previstos.

Neste estudo, foi responsabilizado o grupo de peritos sobre a verificação da validade de conteúdo, o qual elaborou uma tradução consensual adaptada à realidade moçambicana, sem perder a equivalência semântica, conceitual, idiomática e experiencial da escala original (BEATON et al., 2007; FERREIRA et al., 2014; RAMOS, 2014; REICHENHEIM; MORAES, 2007). A este respeito, o consenso do grupo de peritos esteve fundamentado a partir dos dados analisados provenientes dos documentos produzidos durante o processo, assim como estudos relacionados à tradução e contextualização de instrumentos.

Todavia, alguns autores (BEATON et al., 2007; BRACCIALLI et al., 2019a; SOARES; ASSUNÇÃO; SOARES, 2012; SILVA, 2019), consideram que o grupo de especialistas tem a capacidade de modificar as instruções ou o formato do questionário. Em consequência, foi alterado o formato através da inclusão de linhas de separação entre itens, da mesma forma que tem acontecido noutras adaptações da escala MAS (RADLIŃSKA et al., 2020). Igualmente, foi necessário alterar as instruções através da introdução de texto relacionado à maneira de responder o instrumento.

Em relação ao perfil dos participantes durante ambos pré-testes, foram selecionados sujeitos com características semelhantes aos estudos de validação do instrumento, sendo escolhidas pessoas sem deficiência, de ambos os sexos, estudantes dos últimos anos do ensino secundário e universitários (FINDLER; VILCHINSKY; WERNER, 2007; KIM; LU; ESTRADA-HERNANDEZ, 2015; RADLIŃSKA et al., 2020; VILCHINSKY; WERNER; FINDLER, 2010; WÖHRLE; FRANKE; KISSGEN, 2018; e outros)

Deve-se ter em conta que os passos realizados durante o processo de adaptação transcultural fornecem qualidade em relação à validade de conteúdo. Portanto, pode-se concluir que a versão resultante terá confiabilidade e validade se a versão original as tiver (FERREIRA et al., 2014; REICHENHEIM; MORAES, 2007). No entanto, como indica Beaton et al. (2007) esse nem sempre é o caso, talvez devido a diferenças sutis entre as diferentes culturas que tornam um item mais ou menos difícil em relação a outros itens do questionário: tais alterações podem modificar as propriedades estatísticas de um instrumento. É neste sentido, que à semelhança com outras versões do instrumento (KIM; LU; ESTRADA-HERNANDEZ, 2015; RADLIŃSKA et al., 2020; WÖHRLE; FRANKE; KISSGEN, 2018; e outros) foi decidido adicionar as siglas "PT" (MAS-PT) à versão produzida em língua portuguesa, assumindo qualquer viés de tradução e adaptação sobre a escala original. Contudo, como sugerido por Mendonça et al. (2018) foi preparado um segundo estudo relativo a testes adicionais para avaliação das propriedades psicométricas da escala como continuação do processo de adaptação cultural. 


\section{CONCLUSÕES}

A partir dos dados coletados durante a etapa de pré-teste, podemos afirmar que os respondentes compreenderam todo o instrumento, não apresentando dúvidas em relação à linguagem, conceitos ou forma de preenchimento. Neste sentido, julgamos que a escala MAS-PT, traduzida para a língua portuguesa e adaptada ao contexto moçambicano, está pronta para ser usada. Contudo, é recomendável a realização de um processo de avaliação das propriedades psicométricas.

Destaca-se que a disponibilização de uma escala na língua portuguesa, para medir a atitude das pessoas sem deficiência perante as pessoas com deficiência numa perspectiva multidimensional, possibilitará o aumento de pesquisas nesta área contextualizadas em Moçambique.

\section{REFERÊNCIAS}

BEATON, D.; BOMBARDIER, C.; GUILLEMIN, F.; FERRAZ, M. B. Recommendations for the crosscultural adaptation of the DASH \& QuickDASH outcome measures. Institute for Work \& Health, v. 1, n. 1, p. 1-45. 2007.

BERTOLAZI, A. N. Tradução, adaptação cultural e validação de dois instrumentos de avaliação do sono: escala de sonolência de Epworth e índice de qualidade de sono de Pittsburgh. 2008. Dissertação (Mestrado em Ciências Médicas) - Faculdade de Medicina, Universidade Federal do Rio Grande do Sul, Porto Alegre, Brasil, 2008.

BRACCIALli, L. M. P.; BRACCIALli, A. C.; AUDI, M.; SCHERER, M. Tradução e Adaptação Cultural de Instrumentos para Avaliar a Predisposição do Uso de Tecnologia Assistiva que Constitui o Modelo Matching, Person \& Technology. Revista Brasileira de Educação Especial, v. 25 n. 2, p. 189204. 2019a.

BRACCIALLI, L. M. P.; RABADAN, G. B.; MALHEIROS, M. G.; SCHERER, M. Adaptação transcultural para uso no Brasil do Instrumento Survey of Technology Use (SOTU Br). Revista Educação Especial, v. 32, n. 1. 2019 b.

COSTER, W. J.; MANCINI, M. C. Recommendations for translation and cross-cultural adaptation of instruments for occupational therapy research and practice. Revista de Terapia Ocupacional da Universidade de São Paulo, v. 26, n. 1, p. 50-57. 2015.

DORTAS JUNIOR, S. D.; LUPI, O.; DIAS, G. A. C.; GUIMARAES, M. B. S.; VALlE, S. O. R. Adaptação transcultural e validação de questionários na área da saúde. Brazilian Journal Allergy and Immunology, v. 4, n. 1, p. 26-30, 2016.

FERREIRA, L.; NEVES, A. N.; CAMPANA, M. B.; FERNANDES, M. D. C. G. C. Guia da AAOS/IWH: sugestões para adaptação transcultural de escalas. Avaliaçao Psicologica, v. 13, n. 3, p. 457-461, 2014.

FINDLER, L.; VILCHINSKY, N.; WERNER, S. The multidimensional attitudes scale toward persons with disabilities (MAS) construction and validation. Rehabilitation Counseling Bulletin, v. 50, n. 3, p. 166-176, 2007.

FORTES, C. P. D. D.; ARAÚJO, A. P. D. Q. C. Check list para tradução e Adaptação Transcultural de questionários em saúde. Cadernos Saúde Coletiva, v. 27, n. 2, p. 202-209, 2019.

KIM, K. H., LU, J.; ESTRADA-HERNANDEZ, N. Attitudes toward people with disabilities: The tripartite model, social desirability, and other controversial variables. Journal of Asia Pacific Counseling, v. 5, n. 1, p. 23-37, 2015. 
KROPP, J. J.; WOLFE, B. D. College Students' Perceptions on Effects of Volunteering with Adults with Developmental Disabilities. Journal of Higher Education Outreach and Engagement, v. 22, n. 3, p. 93-118, 2018.

LUND, E. M.; SEEKINS, T. Early Exposure to People with Physical and Sensory Disabilities and Later Attitudes toward Social Interactions and Inclusion. Physical Disabilities: Education and Related Services, v. 33, n. 1, p. 1-16, 2014.

MARTÍN, A. R.; ARREGUI, E. A. Development and validation of a scale to identify attitudes towards disability in Higher Education. Psicothema, v. 25, n. 3, p. 370-376, 2013.

MENDONÇA, R. B.; SARNI, R. O. S.; LEN, C.; SOLÉ, D. Tradução para o português (cultura brasileira) e adaptação cultural de questionários para avaliação da qualidade de vida de crianças com alergia alimentar e de seus pais. Brazilian Journal Allergy and Immunology, v. 2, n. 3, p. 364-372, 2018.

MINGANE, E.; RELANDEAU, A.; WAMUSSE, L. (coord.). Síntese do diagnóstico local sobre a situação das pessoas com deficiência em Maputo e Matola. Maputo: Ed. RAVIM \& Handicap International Moçambique, 2010.

MOÇAMBIQUE. Plano Nacional da Área da Deficiência - PNAD II 2012 - 2019. Maputo, set. 2012.

NHAQUILA, H. A. U. Violência Psicológica e Estigmatização dos Estudantes Portadores de Deficiência Auditiva no Ambiente Escolar. 2011. Trabalho de Conclusão de Curso - Faculdade de Letras e Ciências Sociais, Universidade Eduardo Mondlane, Maputo, Moçambique, 2011.

OCETE, C.; PÉREZ-TEJERO, J.; COTERÓN, J. Propuesta de un programa de intervención educativa para facilitar la inclusión de alumnos con discapacidad en educación física. Retos, n. 27, p. 140-145, 2015.

OMOTE, S. Atitudes Sociais em Relação à Inclusão: Recentes Avanços em Pesquisa. Revista Brasileira de Educação Especial, v. 24, n. SPE, p. 21-32, 2018.

PALAD, Y. Y.; BARQUIA, R. B.; DOMINGO, H. C.; FLORES, C. K.; PADILLA, L. I.; RAMEL, J. M. D. Scoping review of instruments measuring attitudes toward disability. Disability and Health Journal, v. 9 , n. 3, p. 354-374, 2016.

RADLIŃSKA， I.; STARKOWSKA， A.; KOŻYBSKA， M.; FLAGA-GIERUSZYŃSKA， K.; KARAKIEWICZ, B. The multidimensional attitudes scale towards persons with disabilities (MAS) - a Polish adaptation (MAS-PL). Annals of Agricultural and Environmental Medicine. 2020. Disponível em: https://doi.org/10.26444/aaem/114531. Acesso em: 10 mar. 2020

RAMOS, A. V. Tradução e adaptação para a cultura portuguesa do Social Touch Questionnaire (STQ). 2014. Dissertação (Mestrado em Fisioterapia) - Escola Superior de Saúde do Alcoitão, Portugal, 2014.

REICHENHEIM, M. E.; MORAES, C. L. Operacionalização de adaptação transcultural de instrumentos de aferição usados em epidemiologia. Revista de Saúde Pública, v. 41, n. 4, p. 665-673, 2007.

SHERRILL, C. Adapted physical activity, recreation, and sport: cross-disciplinary and lifespan. 5. ed. Dubuque: McGraw-Hill, 1998.

SIGAÚQUE, G. A. G. Direito dos deficientes: igualdade de oportunidades. Revista de Direitos Humanos, Maputo, v. 2, n. 2. p. 211-242, 2013.

SILVA, R. Validação da Versão Portuguesa da Multidimensional Attitudes Toward Inclusive Education Scale em Estudantes-Estagiários de Educação Física. 2019. Dissertação (Mestrado em Actividade Física Adaptada) - Faculdade de Desporto da Universidade do Porto, Porto, 2019. 
Luis Rodríguez de Vera Mouliaá; Ângelo José Muria; Ana Belén López Martínez

SOARES, H.; ASSUNÇÃO, V.; SOARES, L. F. Tradução e Validação para português de um questionário de hábitos, conhecimento e atitudes de saúde oral dos adolescentes. Adolescência \& Saúde, Rio de Janeiro, v. 9, n. 1, p 7-11, jan/mar. 2012.

TANAKA, E. D. O. O desenvolvimento de uma escala de atitudes sociais em relação ao trabalho da pessoa com deficiência. 2007. Tese (Doutoramento em Educação) - Faculdade de Filosofia e Ciências, Universidade Estadual Paulista Júlio de Mesquita Filho, Marília, Brasil, 2007.

VILCHINSKY, N.; WERNER, S.; FINDLER, L. Gender and attitudes toward people using wheelchairs: A multidimensional perspective. Rehabilitation Counseling Bulletin, v. 53, n. 3, p. 163-174, 2010.

WÖHRLE, J.; FRANKE, S.; KISSGEN, R. The German Multidimensional Attitude Scale Toward Persons With Disabilities (G-MAS): A factor analytical study among high-school students. Rehabilitation Psychology, v. 63, n. 1, p. 83-97, 2018.

XAVIER, A. D. Inclusão da pessoa com deficiência: a grande barreira são as atitudes. In: CONGRESSO INTERNACIONAL DE EDUCAÇÃO INCLUSIVA, 3., 2016, Natal, Brasil. Anais [...]. Natal, Brasil, 2016.

Recebido em: 21 jun. 2020.

Aprovado em: 29 jul. 2020. 


\title{
Apêndice:
}

\section{Escala Multidimensional de Atitudes perante as pessoas com deficiência (MAS-PT)}

\begin{abstract}
Imagine a seguinte situação: "José/Michela saiu para almoçar a um restaurante com alguns amigos. Um homem/mulher em cadeira de rodas, que José/Michela não conhece, entra no restaurante e junta-se ao grupo. José/Michela é apresentado/a a esta pessoa, e pouco depois, todos os outros vão embora, deixando na mesa apenas o/a José/Michela e o/a homem/mulher de cadeira de rodas. José/Michela deve esperar 15 minutos pela boleia dele/a. Tente imaginar a situação".

As pessoas experimentam uma variedade de emoções quando estão envolvidas numa situação como esta. À direita está uma lista de possíveis emoções, que podem surgir antes, durante e/ou depois desta situação. Por favor, assinale com " $\mathrm{x}$ " em cada uma das 16 linhas para indicar a opção que melhor classifique a probabilidade desta emoção aparecer em José/Michela.
\end{abstract}

\begin{tabular}{llccccc}
\hline Emoção & \multicolumn{5}{c}{ Grau de probabilidade } \\
\cline { 2 - 7 } & $\begin{array}{c}\text { De modo } \\
\text { nenhum }\end{array}$ & & & Muitíssimo \\
\hline 1 & Tensão & 1 & 2 & 3 & 4 & 5 \\
\hline 2 & Estresse & 1 & 2 & 3 & 4 & 5 \\
\hline 3 & Abandono & 1 & 2 & 3 & 4 & 5 \\
\hline 4 & Nervosismo & 1 & 2 & 3 & 4 & 5 \\
\hline 5 & Vergonha & 1 & 2 & 3 & 4 & 5 \\
\hline 6 & Relaxamento & 1 & 2 & 3 & 4 & 5 \\
\hline 7 & Tranquilidade & 1 & 2 & 3 & 4 & 5 \\
\hline 8 & Calma & 1 & 2 & 3 & 4 & 5 \\
\hline 9 & Depressão & 1 & 2 & 3 & 4 & 5 \\
\hline 10 & Medo & 1 & 2 & 3 & 4 & 5 \\
\hline 11 & Chateado & 1 & 2 & 3 & 4 & 5 \\
\hline 12 & Culpado & 1 & 2 & 3 & 4 & 5 \\
\hline 13 & Timidez & 1 & 2 & 3 & 4 & 5 \\
\hline 14 & Pena & 1 & 2 & 3 & 4 & 5 \\
\hline 15 & Desgostado & 1 & 2 & 3 & 4 & 5 \\
\hline 16 & Em alerta & 1 & 2 & 3 & 4 & 5 \\
\hline
\end{tabular}

As pessoas experimentam uma variedade de pensamentos quando envolvidas em situações como esta. A seguir está uma lista de possíveis pensamentos, que podem surgir antes, durante e/ou depois desta situação. Por favor, assinale com " $\mathrm{x}$ " em cada uma das 10 linhas para indicar a opção que melhor classifique a probabilidade deste pensamento aparecer em José/Michela.

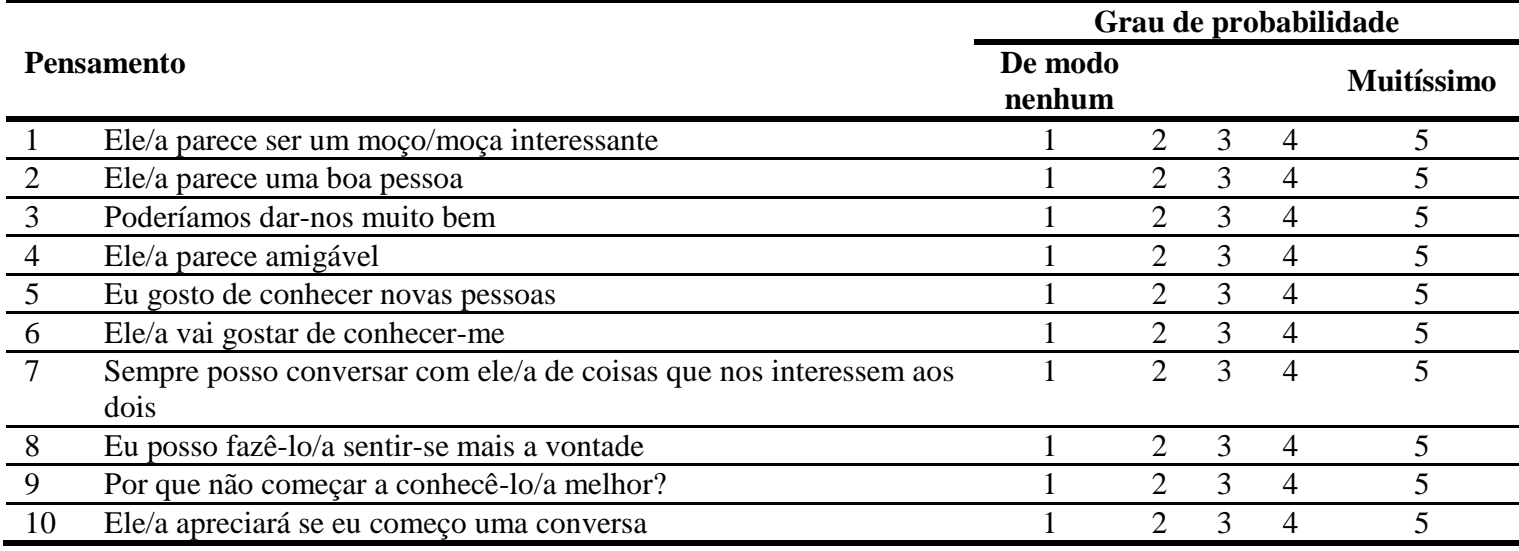

As pessoas experimentam uma variedade de comportamentos quando envolvidas em situações como esta. A seguir está uma lista de possíveis comportamentos, que podem surgir antes, durante e/ou depois desta situação. Por favor, assinale com " $\mathrm{x}$ " em cada uma das 8 linhas para indicar a opção que melhor classifique a probabilidade de José/Michela comportar-se da seguinte maneira.

\begin{tabular}{|c|c|c|c|c|c|}
\hline \multirow[b]{2}{*}{ Comportamento } & \multicolumn{5}{|c|}{ Grau de probabilidade } \\
\hline & $\begin{array}{c}\text { De modo } \\
\text { nenhum }\end{array}$ & & & & Muitíssimo \\
\hline $1 \quad$ Afastar-se & 1 & 2 & 3 & 4 & 5 \\
\hline 2 Levantar-se e sair & 1 & 2 & 3 & 4 & 5 \\
\hline 3 Ler um jornal ou falar ao telefone & 1 & 2 & 3 & 4 & 5 \\
\hline 4 Continuar com aquilo que ele/a estava fazendo & 1 & 2 & 3 & 4 & 5 \\
\hline $\begin{array}{ll}5 & \text { Encontrar uma desculpa para sair } \\
\end{array}$ & 1 & 2 & 3 & 4 & 5 \\
\hline $6 \quad$ Trocar de mesa & 1 & 2 & 3 & 4 & 5 \\
\hline 7 Iniciar uma conversa se ele/a não o faz primeiro & 1 & 2 & 3 & 4 & 5 \\
\hline 8 Começar uma conversa & 1 & 2 & 3 & 4 & 5 \\
\hline
\end{tabular}

